\section{Manifestações neurológicas em ribeirinhos de áreas expostas ao mercúrio na Amazônia brasileira}

\author{
Neurological manifestations in riverine populations \\ from areas exposed to mercury in the \\ Brazilian Amazon
}

\author{
Manifestaciones neurológicas en las comunidades \\ ribereñas expuestas al mercurio de la \\ Amazonia brasileña
}

\author{
Eliana Dirce Torres Khoury 1 \\ Givago da Silva Souza 1 \\ Luiz Carlos de Lima Silveira 1 \\ Carlos Araújo da Costa 1 \\ Amélia A. de Araújo 2 \\ Maria da Conceição Nascimento Pinheiro ${ }^{1}$
}

\footnotetext{
${ }^{1}$ Núcleo de Medicina Tropical, Universidade Federal do Pará, Belém, Brasil.

2 Secretaria Municipal de Saúde de Itaituba, Itaituba Brasil.

Correspondência M. C. N. Pinheiro

Núcleo de Medicina Tropical, Universidade Federal do Pará. Av. Generalíssimo Deodoro 92, Belém, PA 66055-240, Brasil. mconci@ufpa.br
}

\begin{abstract}
This study evaluated current levels of mercury exposure and sensory symptoms in adults from three riverine communities in Pará State, Brazil, two of which located in the Tapajós River basin and one in the Tocantins basin. Participants in this study included 78 residents in Barreiras (Tapajós), 30 in São Luiz do Tapajós (Tapajós), and 49 in Furo do Maracujá (Tocantins). Total hair mercury concentrations were quantified by atomic absorption spectrophotometry, and neurological evaluation was conducted by routine examination. Mercury concentrations in the Tapajós communities were higher than those in the Tocantins $(p<0.01)$. Evaluation of neurological changes showed no significant difference between the communities in exposed areas and control areas for the changes observed by conventional neurological examination, except for gait deviation $(p<0.05)$. The study concludes that despite the mercury exposure levels, there was a low frequency of sensory alterations according to conventional neurological testing.
\end{abstract}

Environmental Exposure; Nervous System Mercury Poisoning; Mercury

\section{Resumo}

No presente estudo, foram avaliados comparativamente os níveis atuais de exposição ao mercúrio e as manifestações neurológicas em ribeirinhos residentes em comunidades situadas no Estado do Pará, Brasil. Participaram do estudo 78 ribeirinhos de Barreiras (bacia do rio Tapajós), 30 de São Luiz do Tapajós (bacia do rio Tapajós) e 49 do Furo do Maracujá (bacia do rio Tocantins). As concentrações de mercúrio total foram quantificadas, em cabelo, pela espectrofotometria de absorção atômica, e a avaliação neurológica foi realizada por meio de testes de rotina. As concentrações de mercúrio nas comunidades do Tapajós foram maiores que as do Tocantins ( $p<0,01)$. A avaliação das alterações neurológicas não mostrou diferença significativa entre as comunidades das áreas expostas e controle para os resultados observados pelo exame neurológico convencional, exceto para desvio da marcha $(p<$ 0,05). Concluiu-se que, apesar dos níveis de exposição ao mercúrio, houve uma baixa frequência de alterações somatossensoriais encontradas por meio de exames neurológicos convencionais.

Exposição Ambiental; Intoxicação do Sistema Nervoso por Mercúrio; Mercúrio 


\section{Introdução}

As intoxicações por mercúrio ocorridas no Japão e no Iraque durante as décadas de 1960 e 1970 causaram, nas pessoas atingidas, um quadro clínico caracterizado por várias alterações sensoriais e motoras 1,2,3. Esses episódios geraram preocupação mundial com o perigo da exposição ao mercúrio, culminando nos relatórios da Organização Mundial da Saúde (OMS) que definiram que níveis de exposição de mercúrio correspondentes a $50 \mu \mathrm{g} / \mathrm{g}$ do cabelo estavam associados a um risco de $5 \%$ de alterações neurológicas em adultos 4,5 .

Os dados que basearam as conclusões da OMS foram todos adquiridos em populações com exposição alta e de curto a médio período temporal. Em diferentes locais do mundo, há exposições ao mercúrio que não se encaixam aos padrões de exposição que levaram às normativas da OMS. Por exemplo, na Amazônia, há uma crônica exposição ao mercúrio em comunidades ribeirinhas que se alimentam principalmente do pescado. Isso ocorre devido à emissão de toneladas de mercúrio no ambiente e que são acumulados por meio da cadeia trófica ${ }^{6,7}$. A exposição humana ao mercúrio pela ingestão de alimentos contaminados é, sem dúvida, a mais importante forma de exposição não ocupacional. Além da Amazônia, esse tipo de exposição tem sido relatado em diferentes acidentes ambientais, com negativos impactos sobre a saúde das populações envolvidas 8,9,10,11. O diagnóstico da intoxicação por mercúrio se baseia em achados epidemiológicos, clínicos e nas concentrações elevadas de mercúrio no sangue ou em amostras de cabelo 12 .

Há evidências que comunidades ribeirinhas da Amazônia brasileira que consomem regularmente peixes na dieta apresentam níveis de exposição ao mercúrio que oferecem riscos à saúde. Os níveis de mercúrio no cabelo de ribeirinhos da bacia do Tapajós têm sido extensivamente acompanhados por diferentes grupos de pesquisa do Brasil e do exterior 13,14,15,16,17,18,19,20, $21,22,23,24,25,26$. Ao longo das últimas duas décadas, essas comunidades têm apresentado concentrações médias de mercúrio no cabelo maiores que aquelas apresentadas por áreas não expostas ao mercúrio, mas abaixo dos níveis que a OMS considera de risco ao sistema nervoso de adultos $(50 \mu \mathrm{g} / \mathrm{g}) 24,26$.

O sistema nervoso é o alvo da ação tóxica do mercúrio, e as manifestações neurológicas devem ser investigadas em indivíduos ou em populações com história de exposição ao mercúrio pela alimentação, principalmente de pescado de áreas contaminadas. Alguns estudos têm ava- liado as consequências da exposição ao mercúrio sobre o funcionamento do sistema nervoso dessas populações ou populações que sofrem de exposição semelhante 27,28,29,30,31,32,33,34,35,36. Outros estudos têm mostrado que populações expostas ao mercúrio pela ingestão de peixes e com índices de mercúrio abaixo dos preconizados pela OMS apresentam alterações neurológicas diversas 27,28,32,34,37,38.

Este estudo tem o objetivo de comparar a frequência de sinais e sintomas neurológicos em ribeirinhos residentes em áreas reconhecidamente expostas ao mercúrio com área não exposta no Estado do Pará.

\section{Material e método}

\section{Caracterização dos sítios}

O estudo foi realizado em comunidades ribeirinhas de duas diferentes regiões do Estado do Pará, sendo duas comunidades localizadas na região da bacia do rio Tapajós, Barreiras e São Luiz do Tapajós, no Município de Itaituba, a qual sofre influência de garimpos de ouro e uma comunidade localizada na região do rio Acará, afluente do baixo Tocantins, no Município de Acará, Furo do Maracujá, na qual não há influência conhecida de atividade garimpeira.

A comunidade de Barreiras, com as coordenadas geográficas $04^{\circ} 05^{\prime} 52^{\prime \prime}$ de latitude sul e 5540'59" de longitude oeste, encontra-se a jusante da cidade de Itaituba e está à margem esquerda do rio Tapajós. De acordo com o levantamento realizado em 2009, pelos agentes comunitários de saúde (ACS) da equipe do Programa Saúde da Família (PSF), a população estimada era de 926 habitantes, em 230 domicílios, sendo o peixe da região a base da alimentação, enquanto a pesca e a agricultura de subsistência eram as atividades ocupacionais mais comuns.

A comunidade de São Luiz do Tapajós, com as coordenadas geográficas $04^{\circ} 20^{\prime} 31^{\prime \prime}$ de latitu-

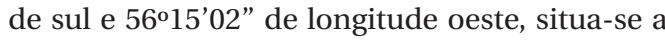
montante da cidade de Itaituba, à margem direita do rio Tapajós. A população residente foi estimada em 620 habitantes, distribuída em 210 domicílios 39. A produção agrícola do povoado resume-se praticamente à mandioca. A base proteica da alimentação é o peixe da região.

A comunidade Furo do Maracujá, com as coordenadas geográficas $01^{\circ} 32^{\prime} 07^{\prime \prime}$ de latitude sul e 48²9'26" de longitude oeste, localiza-se na região do baixo Acará, Município de Acará, pertencente à microrregião de Tomé-Açu, da mesorregião do Nordeste Paraense, que se interligam às bacias dos rios Guamá, Mojú e Capim. A localidade tem 
cerca de 60 residências, representando em torno de 300 habitantes, sendo o consumo do pescado local a principal fonte de proteína na dieta. Não há registros de desmatamentos nessa área, uma vez que a atividade extrativista básica é a colheita de açaí (Euterpe oleracea), tendo, como suporte, a cobertura vegetal do tipo várzea 39 .

\section{População alvo}

Participaram 157 indivíduos, sendo 30 indivíduos de São Luiz do Tapajós, 78 indivíduos de Barreiras e 49 indivíduos do Furo do Maracujá (área controle). As pessoas foram alocadas no estudo considerando-se os seguintes critérios de inclusão: homens e mulheres com idade entre 13 e 53 anos, com residência permanente na comunidade há mais de dois anos. Foram também usados os seguintes critérios de exclusão: diagnóstico confirmado de outras patologias com envolvimento do sistema nervoso; ter exercido atividade ocupacional recente com exposição ao vapor de mercúrio; apresentar dificuldade em prestar informações necessárias para o estudo.

O estudo foi realizado no período de junho de 2011 a junho de 2012. Foram avaliados os níveis de mercúrio em amostras de cabelo, história clínica-epidemiológica e exame neurológico.

\section{Obtenção de amostra de cabelo e análise do mercúrio total}

Colheu-se aproximadamente 10 a $20 \mathrm{mg}$ de cabelo, retirados com tesoura de aço inoxidável, preferentemente da região occipital do couro cabeludo, cerca de $1 \mathrm{~cm}$ acima de sua inserção. As amostras de cabelo foram processadas e analisadas, para determinação de mercúrio total (Hg total), no Laboratório de Toxicologia Humana e Ambiental do Núcleo de Medicina Tropical, da Universidade Federal do Pará (NMT/UFPA), pela espectrofotometria de absorção atômica, usando o Mercury Analyzer modelo HG 201 (Sanso Seisakusho, Tóquio, Japão), utilizando a técnica descrita previamente 14. A qualidade analítica foi garantida pela utilização do padrão do certificado internacional IAEA 085. Todas as amostras foram analisadas em duplicatas, e os resultados, transformados em $\mu \mathrm{g} / \mathrm{g}$.

\section{Hematologia e bioquímica clássicas}

Foram avaliadas as provas hematológicas (hematócrito e dosagem de hemoglobina) e bioquímica (glicemia de jejum, creatinina, GGT, TGO, TGP), em todos os participantes, para auxiliar o diagnóstico de doenças não evidenciáveis ao exame clínico e analisar as interferências com os outros resultados obtidos. Essa etapa foi realizada em trabalho de campo por técnicos do Laboratório de Análises Clínicas do NMT/UFPA, durante as visitas programadas em cada localidade. Os resultados foram avaliados e entregues aos pacientes no momento da consulta médica.

Para obter a percentagem de hemácias no volume total de sangue, foi utilizada a técnica de microematócrito. O sangue foi centrifugado em tubo capilar por 5 minutos a 11.000rpm em centrífuga de microematócrito. Para verificar a lise de hemácias por meio da hemoglobina, foi utilizado o método de reação da cianometahemoglobina. O sangue foi diluído (1:250) em reagente de cor (kit Labtest; Labtest Diagnóstica S.A., Belo Horizonte, Brasil). A leitura foi feita em $\lambda=$ 540nm, no espectrofotômetro semiautomático (BTS-310; Biosystems S.A., Barcelona, Espanha). $\mathrm{O}$ valor obtido foi expresso em $\mathrm{mg} / \mathrm{dL}$.

Para realização de ensaios de bioquímica básica, o soro foi obtido após centrifugação do sangue, sem anticoagulante, por 5 minutos a 3.000rpm. Os ensaios bioquímicos seguiram as seguintes metodologias de acordo com o teste: sistema colorimétrico para a determinação de creatinina em amostras de soro, com reação de ponto final. Aplicação semiautomática utilizando metodologia colorimétrica (picrato alcalino) 40. Utilizou-se um sistema enzimático para a determinação da glicose no sangue em método cinético, com aplicação semiautomática, utilizando a metodologia GOD 41 . O sistema utilizado para medida da atividade das transaminase pirúvica (TGP) e transaminase oxalacética (TGO) foi em amostra de sangue, por método cinético de tempo fixo e medição de ponto final com aplicação semiautomática, utilizando a metodologia colorimétrica 42 . Para a medida de gama glutamil transpeptidase (GGT), utilizouse amostra de soro por fotometria de modo cinético com calibrador proteico.

\section{Exame neurológico convencional}

Cada sujeito foi avaliado por dois médicos. Inicialmente, avaliado por um clínico geral que conduziu um exame neurológico geral preliminar e obteve a história clínica, dados demográficos, tais como idade, sexo, tempo de residência, ocupação, além de informações sobre comorbidades e sinais e sintomas atuais com enfoque para distúrbios somatossensoriais, comprometimento auditivo, visual, gustação, olfato, incoordenação motora, marcha e alterações de movimentos, problemas mentais e intelectuais além de queixas gerais.

Em seguida, foi realizado exame neurológico padrão em todos os sujeitos do estudo por um 
médico neurologista, por meio da semiologia neurológica 43,44,45, com ênfase na avaliação de funções neurológicas comprometidas na intoxicação pelo metilmercúrio. Todos os examinados foram previamente orientados sobre cada teste realizado durante o exame.

A avaliação neurológica específica incluiu: estado mental, para verificar se havia alteração capaz de comprometer a compreensão dos testes; avaliação da fala, para verificar disartria; presença de movimentos involuntários; equilíbrio dinâmico, pela marcha em linha reta e marcha pé ante pé; equilíbrio estático, pela pesquisa do sinal de Romberg e pelo teste de equilíbrio em um só pé (direito e esquerdo), alternadamente; coordenação motora dos membros, pelo teste dedo-nariz e calcanhar-joelho e prova da diadococinesia; força muscular nos segmentos proximais e distais dos membros e musculatura cervical, pelo movimento ativo e movimento contra a resistência, graduada de 0 a 5 conforme a escala de força do Medical Research Council, modificada; reflexos osteotendinosos bicipital, tricipital, estilorradial, patelar e aquileu foram avaliados em ambos os lados, utilizando um martelo de reflexos, sendo graduados de 0 a $4+$; pesquisa do sinal de Babinski.

Para o exame da sensibilidade superficial, utilizou-se pincel de pelos macios próprio para o exame da sensibilidade tátil, e agulha própria para exame da sensibilidade dolorosa. O avaliado era previamente orientado a manter os olhos fechados e dizer "sim" quando percebesse o toque. Posteriormente, eram comparadas as regiões proximais e distais, direita e esquerda, sendo julgada normal ou alterada, e, nesse caso, caracterizada a alteração. O sentido de posição (sensibilidade cinético-postural) foi avaliado nos dedos indicadores e no hálux, de ambos os lados. $\mathrm{O}$ avaliado era mantido em decúbito dorsal, com olhos fechados, e os segmentos, deslocados lenta e suavemente para cima e para baixo, aleatoriamente, a pequena distância e finalmente detido em uma determinada posição, solicitando-se ao examinado identificar a posição do dedo. A avaliação da estereognosia ocorreu pela identificação, com olhos fechados, de uma chave ou moeda colocada em cada mão, alternadamente. A grafestesia foi avaliada pelo reconhecimento, com olhos fechados, de um círculo ou da letra " $\mathrm{x}$ " desenhados em cada mão, alternadamente.

A avaliação dos nervos cranianos incluiu: avaliação da olfação utilizando pó de café e canela quando havia queixa olfatória; avaliação da visão pela análise do fundo de olho e do campo visual pelo método da confrontação, considerando o distúrbio visual presente quando havia $80^{\circ}$ ou menos de visão lateral; avaliação dos nervos motores oculares com a identificação da presença de ptose palpebral; exame dos movimentos oculares (monocular e binocular); avaliação da presença de movimentos de seguimento e de movimentos sacádicos, nistagmo e estrabismo; avaliação das pupilas por meio da análise de simetria e do reflexo fotomotor; avaliação da motricidade da face pela contração da musculatura da face; avaliação da audição por meio da prova de Rinne, a qual avalia as conduções óssea e aérea com o uso de um diapasão de $128 \mathrm{~Hz}$; avaliação do palato e faringe e língua pela inspeção estática e dinâmica.

\section{Análise estatística}

As variáveis qualitativas foram apresentadas por distribuições proporcionais. As variáveis quantitativas foram apresentadas por medidas de tendência central e de variação. A inferência estatística foi realizada pela aplicação de testes de hipótese. O teste do qui-quadrado foi utilizado na comparação das variáveis qualitativas. As variáveis quantitativas foram comparadas pela ANOVA de uma via (nível de significância $=0,05$ ). Todo o processamento estatístico foi realizado no programa BioEstat versão 5.0 (Sociedade Civil Mamirauá, Manaus, Brasil).

\section{Aspectos éticos}

O projeto foi submetido à avaliação do Comitê de Ética em Pesquisa do NMT/UFPA, de acordo com a Resolução no 196/96 do Conselho Nacional de Saúde, com o Protocolo 002/2011-CEP/NMT, aprovado em 24 de março de 2011.

\section{Resultados}

\section{Quantificação dos níveis de Hg total}

As concentrações médias de Hg total nas comunidades da região do Tapajós (áreas contaminada por mercúrio) foram de 8,66 \pm 9,24 $\mu \mathrm{g} / \mathrm{g}$ em Barreiras e de 9,19 $\pm 6,4 \mu \mathrm{g} / \mathrm{g}$ em São Luiz do Tapajós. No Furo do Maracujá (área sem indícios de contaminação por mercúrio), foi de 0,73 $\pm 0,59$. A proporção de indivíduos com níveis de $\mathrm{Hg}$ total no cabelo > 10 $\mu \mathrm{g} / \mathrm{g}$ foi de 33,3\% em São Luiz do Tapajós e de $24,4 \%$ em Barreiras. Nenhum caso acima desse limite foi registrado no Furo do Maracujá. A distribuição das concentrações de Hg total em amostras de cabelo é mostrada na Figura 1. 
Distribuição da concentração de mercúrio total (Hg total) em cabelo de ribeirinhos do Estado do Pará, Brasil, em regiões expostas e não exposta à contaminação por metilmercúrio.

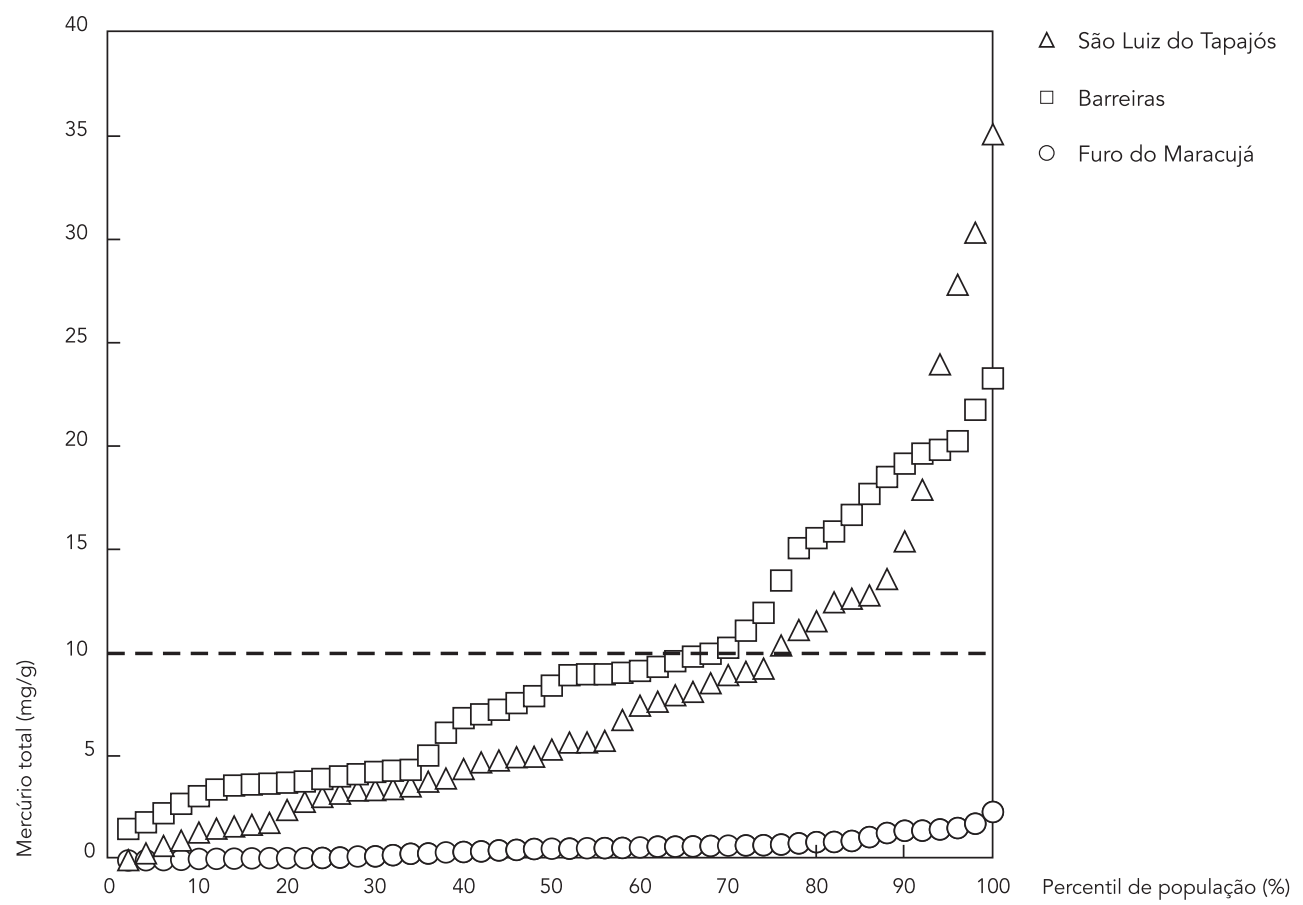

Nota: os símbolos representam a porcentagem das populações que estão abaixo de uma determinada dosagem de mercúrio Para uma mesma parcela da população, as dosagens em São Luiz do Tapajós e Barreiras são sempre superiores às dosagens no Furo do Maracujá. Nas comunidades de São Luiz do Tapajós e Barreiras, houve uma grande parcela da população a

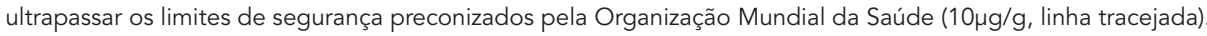

\section{Manifestações clínico-neurológicas}

As queixas relacionadas ao sistema nervoso, quando analisadas como presentes ou ausentes, e a comparação pelo teste do qui-quadrado das proporções entre as duas áreas de exposição ao mercúrio, Barreiras versus São Luiz do Tapajós, não mostraram diferença significativa, exceto para a queixa de fraqueza nas pernas, mais frequente em São Luiz do Tapajós. A comparação entre as áreas de exposição e área controle mostra significância estatística $(\mathrm{p}<0,05)$ para alteração de memória, náuseas/vômitos, adormecimento nas mãos, insônia, tristeza, ansiedade, medo e agressividade, todas mais frequentes na localidade do Furo do Maracujá (Tabela 1).

Pela inspeção, não foi observado tremor ou qualquer outro tipo de movimento involuntário anormal em nenhum dos selecionados para o estudo, tanto na área de exposição como na área de comparação. Disartria também não foi observada.

Na avaliação do equilíbrio, detectou-se alteração da marcha em 3,8\% em Barreiras e 16,7\% em São Luiz do Tapajós, enquanto nenhum caso foi observado no Furo do Maracujá. A comparação por meio do teste do qui-quadrado mostrou haver diferença significativa entre São Luiz do Tapajós e Furo do Maracujá. Nos outros testes do equilíbrio, foi detectada alteração na marcha pé ante pé (marcha tandem) em apenas um caso na localidade de São Luiz do Tapajós, sem significância estatística (Tabela 2).

O exame da coordenação motora mostrou alteração apenas na prova da diadococinesia, caracterizada como incerta em dois casos $(2,6 \%)$ de Barreiras e em dois casos $(6,7 \%)$ de São Luiz do Tapajós, enquanto, nos indivíduos do Furo do Maracujá, não foi detectada alteração. Não foram observadas alterações da coordenação 
Tabela 1

Queixas relacionadas ao sistema nervoso.

\begin{tabular}{|c|c|c|c|c|}
\hline Sintomas & $\begin{array}{c}\text { Barreiras } \\
{[n=78(\%)]}\end{array}$ & $\begin{array}{l}\text { São Luiz do } \\
\text { Tapajós } \\
{[n=30(\%)]}\end{array}$ & $\begin{array}{c}\text { Furo do } \\
\text { Maracujá } \\
{[n=49(\%)]}\end{array}$ & Valor de $p$ \\
\hline Dificuldade para engolir & $6(7,7)$ & $4(13,3)$ & $8(16,3)$ & 0,59 \\
\hline Cefaleia & $65(83,3)$ & $27(90,0)$ & $42(85,7)$ & 0,78 \\
\hline Alteração de memória & $49(62,8)$ & $13(43,3)$ & $31(63,3)$ * & 0,02 * \\
\hline Náuseas/Vômitos & $24(30,8)$ & $3(10,0)$ & $19(38,8)$ * & 0,02 * \\
\hline Adormecimento nas mãos & $28(35,9)$ & $4(13,3)$ & $21(42,9)$ & 0,03 * \\
\hline Adormecimento nos pés & $17(21,8)$ & $3(10,0)$ & $15(30,6)$ & 0,34 \\
\hline Adormecimento perioral & $4(5,1)$ & $1(3,3)$ & $1(2,0)$ & 0,99 \\
\hline Tontura (rotatória) & $47(60,3)$ & $18(60,0)$ & $38(77,6)$ & 0,28 \\
\hline Desequilíbrio para andar & $3(3,8)$ & $0(0,0)$ & $6(12,2)$ & 0,99 \\
\hline Tremor & $16(20,5)$ & $3(10,0)$ & $17(34,7)$ & 0,08 \\
\hline Diminuição da força para prender/segurar & $16(20,5)$ & $4(13,3)$ & $10(20,4)$ & 0,99 \\
\hline Fraqueza nas pernas & $10(12,8)$ & $12(40,0) * *$ & $12(24,5)$ & $0,03 * \star$ \\
\hline Câimbras & $29(37,2)$ & $15(50,0)$ & $30(61,2)$ & 0,12 \\
\hline Dor nos membros & $29(37,2)$ & $15(50,0)$ & $25(51,0)$ & 0,18 \\
\hline Dificuldade visual & $34(43,6)$ & $17(56,7)$ & $26(53,1)$ & 0,58 \\
\hline Dificuldade auditiva & $8(10,3)$ & $2(6,7)$ & $5(10,2)$ & 0,79 \\
\hline Alteração do olfato & $1(1,3)$ & $0(0,0)$ & $0(0,0)$ & 0,99 \\
\hline Insônia & $13(16,7)$ & $7(23,3)$ & 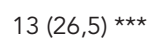 & $0,02 * \star \star *$ \\
\hline Tristeza & $15(19,2)$ & $3(10,0)$ & $18(36,7) \#$ & $<0,01 \#$ \\
\hline Ansiedade & $12(15,4)$ & $4(13,3)$ & $20(40,8) \#$ & $<0,01 \#$ \\
\hline Medo & $13(16,7)$ & $4(13,3)$ & $22(44,9) \#$ & $<0,01 \#$ \\
\hline Depressão & $6(7,7)$ & $1(3,3)$ & $3(6,1) \#$ & 0,86 \\
\hline Agressividade & $0(0,0)$ & $0(0,0)$ & $9(18,4) \#$ & $<0,01 \#$ \\
\hline
\end{tabular}

* São Luiz do Tapajós x Furo do Maracujá;

** Barreiras x São Luiz do Tapajós;

*** Barreiras x Furo do Maracujá;

\# Furo do Maracujá x áreas de exposição.

motora dos membros nas provas dedo-nariz e calcanhar-joelho, assim como no teste para coordenação motora do tronco, nas três comunidades do estudo.

$\mathrm{Na}$ avaliação dos reflexos osteotendinosos, a frequência de hiper-reflexia nos membros superiores foi pequena nas três comunidades do estudo. Nos membros inferiores, a frequência foi um pouco maior e ocorreu principalmente por aumento do reflexo patelar. A comparação estatística da hiper-reflexia nas três localidades não constatou diferença significativa (valor de $\mathrm{p}>0$,05) (Tabela 2).

$\mathrm{Na}$ avaliação dos nervos cranianos, foi observada baixa frequência de alteração. O campo visual avaliado pelo método da confrontação detectou alteração apenas em três dos selecionados de Barreiras e em um em São Luiz do Tapajós. A audição avaliada pela prova de Rinne foi o exame de nervos cranianos que detectou maior frequência de alteração de nervos cranianos. A comparação das frequências das alterações dos nervos cranianos nas três comunidades, realizada pelo teste do qui-quadrado, não mostrou diferença estatística significante (Tabela 2).

$\mathrm{Na}$ avaliação da sensibilidade superficial, constatou-se diminuição da sensibilidade (hipoestesia) tanto tátil como dolorosa em 9 (11,5\%) dos ribeirinhos residentes em Barreiras, 4 $(13,3 \%)$ em São Luiz do Tapajós e 1 (2\%) no Furo do Maracujá. Na localidade de Barreiras, foram encontrados diversos tipos de distribuição da hipoestesia: meia, luva, meia e luva, e hemihipoestesia. Em São Luiz do Tapajós, observou-se somente distribuição em meia e luva bilateral e em meia. No Furo do Maracujá, o único caso em que a hipoestesia era tanto tátil como dolorosa mostrava distribuição em meia unilateral. Nos 
Frequência de alterações neurológicas nas três comunidades.

\begin{tabular}{|c|c|c|c|c|}
\hline & $\begin{array}{c}\text { Barreiras } \\
{[n=78(\%)]}\end{array}$ & $\begin{array}{l}\text { São Luiz do } \\
\text { Tapajós } \\
{[n=30(\%)]}\end{array}$ & $\begin{array}{c}\text { Furo do } \\
\text { Maracujá } \\
{[n=49(\%)]}\end{array}$ & Valor de $\mathrm{p}$ \\
\hline \multicolumn{5}{|l|}{ Equilíbrio } \\
\hline Marcha alterada & $3(3,8)$ & $5(16,7)$ * & $0(0,0)$ & $<0,01$ \\
\hline Marcha pé ante pé instável & $0(0,0)$ & $1(3,3)$ & $0(0,0)$ & 0,6 \\
\hline Sinal de Romberg & $0(0,0)$ & $0(0,0)$ & $0(0,0)$ & Não disponível \\
\hline Equilíbrio em um só pé & $0(0,0)$ & $0(0,0)$ & $0(0,0)$ & Não disponível \\
\hline \multicolumn{5}{|l|}{ Reflexos } \\
\hline \multicolumn{5}{|c|}{ Reflexos osteotendinosos dos membros superiores } \\
\hline Hiper-reflexia & $6(7,7)$ & $3(10,0)$ & $1(2,0)$ & 0,3 \\
\hline Hiporreflexia & $0(0,0)$ & $0(0,0)$ & $0(0,0)$ & Não disponível \\
\hline \multicolumn{5}{|c|}{ Reflexos osteotendinosos dos membros inferiores } \\
\hline Hiper-reflexia & $13(16,7)$ & $7(23,3)$ & $6(12,2)$ & 0,44 \\
\hline Hiporreflexia & $2(2,6)$ & $0(0,0)$ & $0(0,0)$ & 0,36 \\
\hline Reflexo cutâneo plantar & $0(0,0)$ & $0(0,0)$ & $0(0,0)$ & Não disponível \\
\hline \multicolumn{5}{|l|}{ Nervos cranianos } \\
\hline Anosmia & $1(1,3)$ & $0(0,0)$ & $0(0,0)$ & 0,6 \\
\hline \multicolumn{5}{|l|}{ Alteração do campo visual } \\
\hline Unilateral & $3(3,8)$ & $0(0,0)$ & $0(0,0)$ & 0,21 \\
\hline Bilateral & $0(0,0)$ & $1(3,3)$ & $0(0,0)$ & 0,6 \\
\hline \multicolumn{5}{|l|}{ Alteração da audição } \\
\hline Unilateral & $5(6,4)$ & $1(3,3)$ & $1(2,0)$ & 0,48 \\
\hline Bilateral & $2(2,6)$ & $1(3,3)$ & $0(0,0)$ & 0,48 \\
\hline Nistagmo & $1(1,3)$ & $0(0,0)$ & $0(0,0)$ & 0,6 \\
\hline Estrabismo & $1(1,3)$ & $0(0,0)$ & $0(0,0)$ & 0,6 \\
\hline
\end{tabular}

* Valor de $p=0,003$

outros três casos, apenas a sensibilidade dolorosa estava alterada. Não houve diferença significativa entre os grupos estudados (Tabela 3).

A sensibilidade especial avaliada por meio da estereognosia e da grafestesia estava normal na maioria dos avaliados. A agrafestesia foi detectada apenas em dois indivíduos em Barreiras e em um em São Luiz do Tapajós. Não houve significância estatística quando foram comparados pelo teste do qui-quadrado ( $p>0,05)$ (Tabela 3 ).

\section{Discussão}

No presente estudo, os níveis de mercúrio nas amostras de cabelo apresentadas por comunidades ribeirinhas amazônicas expostas ao metal apresentam valores abaixo dos níveis que a OMS considera de risco para alterações funcionais do sistema nervoso (50 $\mu \mathrm{g} / \mathrm{g})$. Apesar disso, vários trabalhos têm identificado que sujeitos dessas comunidades apresentam algum tipo de perda sensório-motora ou neuropsicológica $27,28,32,34,37,38$. As alterações neurológicas encontradas nesses estudos, em sua maioria, são procedimentos experimentais menos convencionais da prática neurológica de rotina, como visão de cores, visão de contraste e baterias neuropsicológicas.

\section{Quantificação dos níveis de Hg total}

As comunidades de Barreiras e de São Luiz do Tapajós já foram monitoradas quanto à dosagem de mercúrio em amostra de cabelo de seus residentes, sendo encontrado, como resultado, que os níveis de mercúrio podem ser moderados a elevados 13,14,15,16,17,18,19,20 . No presente estudo, como já era esperado, as concentrações de Hg total foram significativamente maiores nas comunidades da bacia do rio Tapajós, ou seja, nas áreas de exposição ao mercúrio, do que na área controle do Furo do Maracujá, com média de $8,66 \pm 9,24 \mu \mathrm{g} / \mathrm{g}$ em Barreiras e de 9,19 $\pm 6,4 \mu \mathrm{g} / \mathrm{g}$ 
Tabela 3

\begin{tabular}{|c|c|c|c|c|}
\hline Sensibilidade superficial & $\begin{array}{c}\text { Barreiras } \\
{[n=78(\%)]}\end{array}$ & $\begin{array}{l}\text { São Luiz do } \\
\text { Tapajós } \\
\text { [n = } 30(\%)]\end{array}$ & $\begin{array}{l}\text { Furo do Maracujá } \\
\qquad[n=49(\%)]\end{array}$ & Valor de $\mathrm{p}$ \\
\hline \multicolumn{5}{|l|}{ Sensibilidade dolorosa } \\
\hline Normal & $69(88,5)$ & $26(86,7)$ & $45(91,8)$ & \\
\hline Alteração em meia & $2(2,6)$ & $1(3,3)$ & $2(4,1)$ & \\
\hline Alteração em luva & $1(1,3)$ & $0(0,0)$ & $0(0,0)$ & \\
\hline Alteração em meia e luva & $4(5,1)$ & $3(10,0)$ & $2(4,1)$ & \\
\hline Hemihipoestesia & $2(2,6)$ & $0(0,0)$ & $0(0,0)$ & \\
\hline Total de casos alterados & $9(11,5)$ & $4(13,3)$ & $4(8,2)$ & 0,7422 \\
\hline \multicolumn{5}{|l|}{ Sensibilidade tátil } \\
\hline Normal & $69(88,5)$ & $26(86,7)$ & $48(98,0)$ & \\
\hline Alteração em meia & $2(2,6)$ & $1(3,3)$ & $1(2,0)$ & \\
\hline Alteração em luva & $1(1,3)$ & $0(0,0)$ & $0(0,0)$ & \\
\hline Alteração em meia e luva & $4(5,1)$ & $3(10,0)$ & $0(0,0)$ & \\
\hline Hemihipoestesia & $2(2,6)$ & $0(0,0)$ & $0(0,0)$ & \\
\hline Total de casos alterados & $9(11,5)$ & $4(13,3)$ & $1(2,0)$ & 0,1205 \\
\hline \multicolumn{5}{|l|}{ Sensibilidade cinético-postural } \\
\hline Normal & $77(98,7)$ & $30(100,0)$ & $49(100,0)$ & \\
\hline Alterada & $1(1,3)$ & $0(0,0)$ & $0(0,0)$ & 0,6007 \\
\hline \multicolumn{5}{|l|}{ Sensibilidade especial } \\
\hline \multicolumn{5}{|l|}{ Estereognosia } \\
\hline Normal & $78(100,0)$ & $30(100,0)$ & $49(100,0)$ & \\
\hline Alterada & $0(0,0)$ & $0(0,0)$ & $0(0,0)$ & \\
\hline \multicolumn{5}{|l|}{ Grafestesia } \\
\hline Normal & $76(97,4)$ & $29(96,7)$ & $49(100,0)$ & \\
\hline Alterada & $2(2,6)$ & $1(3,3)$ & $0(0,0)$ & 0,4829 \\
\hline
\end{tabular}

em São Luis do Tapajós. A maior concentração de Hg total no cabelo na área contaminada foi de $60 \mu \mathrm{g} / \mathrm{g}$, observada em um pescador residente em Barreiras. Enquanto, na área não exposta, foi de $2,4 \mu \mathrm{g} / \mathrm{g}$.

\section{Avaliação clínica neurológica dos ribeirinhos amazônicos: manifestações subjetivas}

As recomendações da OMS foram baseadas nos surtos com exposição muito elevada, que resultaram em concentrações de mercúrio em cabelo variando em centena de micrograma por grama 37. Entretanto, o perfil dos efeitos da exposição de longo prazo a baixas doses de metilmercúrio permanece indefinido 46 .

Cefaleia, fadiga, tontura, náuseas/vômitos, adormecimento nas mãos, parestesias, alteração de memória, insônia, tristeza, ansiedade, medo e agressividade foram relatados como sintomas comuns na intoxicação por mercúrio. Neste es- tudo, essas manifestações foram mais frequentes na localidade do Furo do Maracujá (área controle) do que nas áreas expostas, sugerindo outras causas que não o mercúrio.

Trabalho anterior 47 descreveu sintomas subjetivos de dormência, vertigem, tontura, cefaleia, dor nas extremidades dos membros e nas costas redução da visão, tremor, irritabilidade, diminuição da audição, perda de memória, fraqueza muscular e insônia relatados por ribeirinhos da bacia do Tapajós que haviam apresentado concentrações de $\mathrm{Hg}$ total no cabelo acima de $20 \mu \mathrm{g} / \mathrm{g}$, admitindo que os sintomas neurológicos relatados não eram específicos, porém, eram semelhantes àqueles observados na doença de Minamata, no Japão. 
Avaliação clínica neurológica dos ribeirinhos amazônicos: exame neurológico convencional

No presente estudo, no exame neurológico convencional, não foram observados, em nenhum dos grupos estudados, o tremor ou qualquer outro movimento involuntário e disartria. Essas manifestações, particularmente o tremor, foram apenas raramente observadas na exposição aguda ocorrida no Iraque 1 . Alguns autores consideram que o tremor pode ser usado como uma medida de exposição crônica a baixos níveis de exposição ao metilmercúrio ${ }^{48}$. Os resultados do presente estudo são discordantes dos resultados de Harada et al. 21, que detectaram tremor, disartria e alteração na coordenação motora. Essas diferenças podem ser decorrentes dos critérios utilizados, uma vez que aquele estudo avaliou clinicamente apenas indivíduos que haviam apresentado níveis de $\mathrm{Hg}$ total no cabelo acima de $20 \mu \mathrm{g} / \mathrm{g}$. O tempo de exposição baseado no tempo de residência local (maior que dois anos) foi uma das exigências para participação no estudo.

Dentre os distúrbios relacionados ao equilíbrio, a marcha alterada, caracterizada por desvio com olhos fechados, foi observada apenas nas comunidades expostas, sendo mais frequente em São Luiz do Tapajós (16,7\%). Entretanto, não foram observadas as alterações do equilíbrio relacionadas aos efeitos neurotóxicos do metilmercúrio como alargamento da base de sustentação e ataxia da marcha. Lebel et al. 28 , utilizando o teste de movimentos alternados de Branches (BAMT), encontraram alta prevalência de movimento desorganizado nos membros $(53,4 \%)$, relacionada a níveis de mercúrio abaixo de $50 \mu \mathrm{g} / \mathrm{g}$, em moradores de Brasília Legal, na bacia do Tapajós. Entretanto, não detectaram alteração significativa da coordenação motora nos testes dedo-nariz e calcanhar-joelho, utilizados no exame neurológico convencional.

$\mathrm{Na}$ avaliação dos reflexos osteotendinosos (profundos), embora a hiper-reflexia nos membros superiores e inferiores tenha sido relativamente frequente nas áreas expostas, não houve diferença significativa entre as três comunidades estudadas. Nos acidentes ambientais ocorridos no Japão e no Iraque, a hiper-reflexia foi mais frequente que a hiporreflexia na fase aguda da doença 49.

As alterações nos reflexos osteotendinosos, com hiper-reflexia, principalmente do reflexo patelar que foram detectadas neste estudo em $16,7 \%$ dos casos de Barreiras e $23,3 \%$ dos casos de São Luiz do Tapajós, estão em concordância com outros estudos realizados na bacia do Tapajós mostrando prevalência maior de hiper-reflexia em relação à hiporreflexia em membros superio- res e inferiores 28,49,50. Entretanto, no estudo de Lebel et al. 28, a hiper-reflexia patelar e bicipital foi encontrada em $44,8 \%$ e $25,9 \%$ dos casos, respectivamente, frequência superior à encontrada neste estudo.

Admite-se que o primeiro sintoma na intoxicação por mercúrio é o distúrbio sensorial caracterizado por parestesia ou disestesia nas extremidades que ocorre mesmo em casos leves 51. A principal causa do distúrbio somatossensorial na doença de Minamata é a lesão do córtex cerebral, e, tal como ocorre nas neuropatias periféricas, $o$ sintoma inicial pode ser distúrbio sensitivo superficial do tipo luva e meia 52 . Cada modalidade de percepção sensorial, incluindo leve toque, dor, sensibilidade ao calor e frio, vibração e posição, pode ser perturbada 51. A hipoestesia tanto tátil como dolorosa foram as alterações de sensibilidade observadas neste estudo, particularmente em ribeirinhos do Tapajós, sendo 11,5\% em Barreiras e 13,3\% em São Luiz do Tapajós.

Na doença de Minamata, foram observados vários tipos de distribuição do distúrbio sensorial: generalizado, hemilateral, vertebral, irregular e incerto, e o tipo em meia e luva, que foi o mais frequente 53 . Neste estudo, a distribuição meia e luva ocorreu em 5,1\% dos casos de Barreiras e em $10 \%$ dos casos de São Luiz do Tapajós, discordando dos resultados de Harada et al. 21 que observaram alteração sensorial em $32 \%$ dos indivíduos estudados na bacia do Tapajós, sendo que $14 \%$ apresentavam distribuição do tipo meia e luva relacionado à concentração de $\mathrm{Hg}$ total no cabelo acima de $20 \mu \mathrm{g} / \mathrm{g}$. Em outro estudo realizado por Harada et al. 12, em uma reserva indígena na província de Ontário, no Canadá, cuja contaminação ocorreu pela cadeia alimentar, os sintomas sensoriais foram os mais proeminentes, sendo detectados muitos casos de alteração de sensibilidade com distribuição em meia e luva.

O distúrbio sensorial se estendendo para as regiões proximais e mostrando um padrão tipo luva e meia é um dos achados característicos da intoxicação por metilmercúrio, apesar de não ser exclusivo dessa 11. Na tragédia de Minamata, a alteração somestésica mostrou distribuição variável, mas o tipo luva e bota foi relativamente frequente.

O exame da sensibilidade especial pela estereognosia constatou poucos casos de alteração nas localidades estudadas. A grafestesia estava normal em 97,4\% dos indivíduos em Barreiras, em 96,7\% em São Luiz do Tapajós e em 100\% no Furo do Maracujá. A alteração caracterizada por agrafestesia, observada em um caso $(3,3 \%)$ em São Luiz do Tapajós, estava relacionada à concentração de Hg total no cabelo acima de 
$10 \mu \mathrm{g} / \mathrm{g}$, e, nos dois casos (2,6\%) em Barreiras, não havia associação com as concentrações atuais de $\mathrm{Hg}$ total maior que $10 \mu \mathrm{g} / \mathrm{g}$. Entretanto, um dos casos tinha história prévia de concentração maior que $10 \mu \mathrm{g} / \mathrm{g}$. Apesar de haver sido observada alteração da estereognosia nas duas grandes tragédias de envenenamento por metilmercúrio que ocorreram no Iraque e no Japão 1,53, na maioria dos estudos que abordaram a exposição ao metilmercúrio, não foram relatadas alterações significativas da grafestesia e da estereognosia.

Os ribeirinhos do Tapajós avaliados no corrente estudo possuíam histórico de exposição prolongada a baixas concentrações de mercúrio, via ingestão de pescado da região, e a média de idade das pessoas afetadas situava-se na faixa etária de trinta anos. As alterações neurológicas estudadas no presente estudo foram importantes para o diagnóstico da intoxicação nos acidentes de Minamata e do Iraque. A avaliação da resposta ao estímulo sensorial é sempre difícil, pois de- pende da interpretação de experiências sensoriais do paciente, e essa, por sua vez, depende da consciência, responsividade geral e capacidade para colaborar, assim como da inteligência, educação e sugestibilidade 54 . Sendo assim, métodos mais elaborados que os rotineiramente usados na clínica médica devem ser necessários para avaliação da função sensorial dos sujeitos expostos ao metal.

Concluiu-se que as alterações neurológicas encontradas nas áreas expostas ao mercúrio sugerem a necessidade de aplicação de técnicas quantitativas para avaliação das funções somatossensoriais, bem como o monitoramento clínico e toxicológico periódico, tendo em vista a possibilidade do diagnóstico de casos leves de intoxicação por mercúrio em comunidades ribeirinhas do Tapajós. Políticas públicas de educação alimentar e de monitoramento dos níveis de mercúrio poderiam auxiliar na prevenção de acometimentos neurológicos em populações expostas ao metal.

\section{Resumen}

En el presente estudio se evaluó comparativamente los niveles actuales de exposición al mercurio y sus manifestaciones neurológicas en los residentes de las comunidades ribereñas en el Estado de Pará, Brasil. Los participantes fueron 78 de las riberas limítrofes (cuenca del Tapajós), 30 en São Luís do Tapajós (cuenca del Tapajós) y 49 en Pasiflora (cuenca del río Tocantins). Las concentraciones de mercurio total en el pelo se cuantificaron por espectrofotometría de absorción atómica y la evaluación neurológica se realizó mediante el análisis de rutina. Las concentraciones de mercurio en las comunidades Tapajós fueron más altas que en Tocantins $(p<0,01)$. La evaluación de los trastornos neurológicos no mostró diferencias significativas entre las comunidades de las zonas expuestas, ni en el control de los resultados observados durante el examen neurológico convencional, excepto por desviaciones de la marcha $(p<0,05)$. Se concluyó que, si bien existen niveles de exposición al mercurio, hubo una baja frecuencia de cambios, cuyo examen neurológico fue de tipo estándar somatosensorial.

\section{Colaboradores}

E. D. T. Khoury colaborou na aquisição de dados, análise de dados, preparação do manuscrito. G. S. Souza, C. A. Costa e A. A. Araújo contribuíram na aquisição de dados, análise de dados, preparação do manuscrito e avaliação final do manuscrito. L. C. L. Silveira participou da concepção da pesquisa, preparação do manuscrito e avaliação final do manuscrito. M. C. N. Pinheiro participou da concepção do trabalho, aquisição dos dados, preparação e avaliação final do manuscrito.

\section{Agradecimentos}

Este trabalho foi financiado pela JICA, CNPq-PRONEX/ FAPESPA/UFPA/FADESP (\#268/2007), CNPq (\#620037/2008-3), CNPq (\#476744/2009-1), CNPqPRONEX/FAPESPA (\#316799/2009), Capes-PROCAD (\#0019/01) e PPSUS/CNPq/FAPESPA. Este grupo de pesquisa é parte da Rede Instituto Brasileiro de Neurociência (IBN Net), sendo financiado pela FINEP. L. C. L. Silveira é bolsista de produtividade do CNPq. 
1. Bakir F, Damluji SF, Amin-Zaki L, Murtadha M Khalidi A, Al-Rawi NY, et al. Methylmercury poisoning in Iraq. Science 1973; 181:230-41.

2. Harada M. Minamata disease: methylmercury poisoning in Japan caused by environmental pollution. Crit Rev Toxicol 1995; 25:1-24.

3. Tsubaki T. Organic mercurial poisoning along the River Agano. Naika 1968; 21:871-5.

4. World Health Organization. Mercury. Geneva: World Health Organization; 1976. (Environmental Health Criteria, 1).

5. World Health Organization. Methylmercury. Geneva: World Health Organization; 1990. (Environmental Health Criteria, 101).

6. Malm O. Gold mining as a source of mercury exposure in the Brazilian Amazon. Environ Res 1998; 77:73-8.

7. Telmer K, Costa M, Simões AR, Araujo ES, Maurice $Y$. The source and fate of sediment and mercury in the Tapajós River, Pará, Brazilian Amazon: Groundand space-based evidence. J Environ Manage 2006; 81:101-13

8. Bakir F, Rustam H, Tikrit S, Al-Damluji SF, Shihristani H. Clinical and epidemiological aspects of methylmercury poisoning. Postgrad Med J 1980; 56:1-10.

9. Soong TR, Chu FR, Fun ZT. Epidemiological study on the health of residents along the Sunhua river, polluted by methylmercury. Environ Sci 1994; 3:41-54.

10. Wheatley B. Exposure of Canadian aboriginal peoples to methylmercury. Environ Sci 1994; 3:33-40.

11. Harada M. Minamata disease. Tokyo: Kumamoto Nichinichi Shinbun Culture \& Information Center; 2004.

12. Harada M, Fujino T, Oorui T, Nakachi S, Nou T, Kizaki T, et al. Follow-up study of mercury pollution in indigenous tribe reservations in the province of Ontario, Canada, 1975-2002. Bull Environ Contam Toxicol 2005; 74:689-97.

13. Akagi H, Malm O, Branches F, Kinjo Y, Kashima Y, Guimarães J, et al. Human exposure to mercury due to gold mining in the Tapajos River Basin, Amazon, Brazil: speciation of mercury in human hair, blood and urine. Water Air Soil Poll 1995; 80:85.

14. Malm O, Branches FJ, Akagi H, Castro MB, Pfeiffer WC, Harada M, et al. Mercury and methylmercury in fish and human hair from the Tapajós river basin, Brazil. Sci Total Environ 1995; 175:141-50.

15. Bidone ED, Castilhos ZC, Cid de Souza TM, Lacerda LD. Fish contamination and human exposure to mercury in the Tapajós River Basin, Pará State, Amazon, Brazil: a screening approach. Bull Environ Contam Toxicol 1997; 59:194-201.

16. Castilhos ZC, Bidone ED, Lacerda LD. Increase of the background human exposure to mercury through fish consumption due to gold mining at the Tapajós River region, Pará State, Amazon. Bull Environ Contam Toxicol 1998; 61:202-9.

17. Akagi H, Naganuma A. Human exposure to mercury and the accumulation of methylmercury that is associated with gold mining in the Amazon Basin, Brazil. J Health Sci 2000; 46:323-8.
18. Santos EC, Jesus IM, Brabo ES, Loureiro EC, Mascarenhas AF, Weirich J, et al. Mercury exposures in riverside Amazon communities in Pará, Brazil. Environ Res 2000; 84:100-7.

19. Santos LS, Müller RC, Sarkis JE, Alves CN, Brabo ES, Santos EO, et al. Evaluation of total mercury concentrations in fish consumed in the municipality of Itaituba, Tapajós River Basin, Pará, Brazil. Sci Total Environ 2000; 261:1-8.

20. Pinheiro M, Nakanishi J, Oikawa T, Guimarães G, Quaresma M, Cardoso B, et al. Methylmercury human exposure in riverside villages of Tapajos basin, Pará State, Brazil. Rev Soc Bras Med Trop 2000; 33:265-9.

21. Harada M, Nakanishi J, Yasoda E, Pinheiro MCN, Oikawa T, Guimarães GA, et al. Mercury pollution in the Tapajós River Basin, Amazon: mercury level of head hair and health effects. Environ Int 2001; 27:285-90

22. Pinheiro MCN, Oikawa T, Vieira JLF, Gomes MSV, Guimarães GA, Crespo-López ME, et al. Comparative study of human exposure to mercury in riverside communities in the Amazon region. Braz J Med Biol Res 2006; 39:411-4.

23. Pinheiro MCN, Macchi BM, Vieira JLF, Oikawa T, Amoras WW, Guimarães GA, et al. Mercury exposure and antioxidant defenses in women: a comparative study in the Amazon region. Environ Res 2008; 107:53-9.

24. Barbieri FL, Gardon J. Hair mercury levels in Amazonian populations: spatial distribution and trends. Int J Health Geogr 2009; 8:71.

25. Berzas Nevado JJ, Rodríguez Martín-Doimeadios RC, Guzmán Bernardo FJ, Jiménez Moreno M, Herculano AM, do Nascimento JL, et al. Mercury in the Tapajós River basin, Brazilian Amazon: a review. Environ Int 2010; 36:593-608.

26. Pinheiro MCN, Farripas SSM, Oikawa T, Costa CA, Amoras WW, Vieira JLF, et al. Temporal Evolution of exposure to mercury in riverside communities in the Tapajós Basin, from 1994 to 2010. Bull Environ Contam Toxicol 2012; 89:119-24.

27. Lebel J, Mergler D, Lucotte M, Amorim M, Dolbec J, Miranda D, et al. Evidence of early nervous system dysfunction in Amazonian populations exposed to low-levels of methylmercury. Neurotoxicology 1996; 17:157-67.

28. Lebel J, Mergler D, Branches F, Lucotte M, Amorim M, Larribe F, et al. Neurotoxic effects of low-level methylmercury contamination in the Amazonian Basin. Environ Res 1998; 79:20-32.

29. Grandjean P, White RF, Nielsen A, Cleary D, Santos ECO. Methylmercury neurotoxicity in Amazonian children downstream from gold mining. Environ Health Perspect 1999; 107:587-91.

30. Dolbec J, Mergler D, Passos CJS, Morais SS, Lebel J. Methylmercury exposure affects motor performance of a riverine population of the Tapajós river, Brazilian Amazon. Int Arch Occup Environ Health 2000; 73:195-203.

31. Mergler D. Review of neurobehavioral deficits and river fish consumption from the Tapajós (Brazil) and St. Lawrence (Canada). Environ Toxicol Pharmacol 2002; 12:93-9. 
32. Silveira L, Damin E, Pinheiro M, Rodrigues A, Moura A, Côrtes M, et al. Visual dysfunction following mercury exposure by breathing mercury vapour or by eating mercury-contaminated food. In: Mollon J, Pokorny J, Knoblauch K, editors. Normal and defective colour vision. Oxford: Oxford University Press; 2003. p. 407-17.

33. Tavares LMB, Câmara VM, Malm O, Santos ECO. Performance on neurological development tests by riverine children with moderate mercury exposure in Amazonia, Brazil. Cad Saúde Pública 2005; 21:1160-7.

34. Rodrigues AR, Souza CRB, Braga AM, Rodrigues PSS, Silveira AT, Damin ETB, et al. Mercury toxicity in the Amazon: contrast sensitivity and color discrimination of subjects exposed to mercury. Braz J Med Biol Res 2007; 40:415-24.

35. Fillion M, Lemire M, Philibert A, Frenette B, Weiler HA, Deguire JR, et al. Visual acuity in fish consumers of the Brazilian Amazon: risks and benefits from local diet. Public Health Nutr 2011; 14:2236-44.

36. Fillion M, Philibert A, Mertens F, Lemire M, Passos CJ, Frenette B, et al. Neurotoxic sequelae of mercury exposure: an intervention and follow-up study in the Brazilian Amazon. Ecohealth 2011; 8:210-22.

37. Maruyama K, Yorifuji T, Tsuda T, Sekikawa T, Nakadaira H, Saito H. Methylmercury exposure at Niigata, Japan: results of neurological examinations of 103 adults. J Biomed Biotechnol 2012; 2012:635075.

38. Yorifuji T, Tsuda T, Takao S, Suzuki E, Harada M. Total mercury content in hair and neurologic signs: historic data from Minamata. Epidemiology 2009; 20:188-93

39. Amoras WW. Interferentes bio-sócio-ambientais na exposição ao mercúrio em crianças ribeirinhas de diferentes regiões da Amazônia [Tese de Doutorado]. Belém: Núcleo de Medicina Tropical, Universidade Federal do Pará; 2011.

40. Jaffe M. Ueber den Niederschlag welchen Pikrinsäure in normalen Harn erzeugt und über eine neue reaction des Kreatinins. Z Physiol Chem 1886; 10:391-400.

41. Trinder P. Determination of glucose in blood using glucose oxidase with an alternative oxygen aceptor. Ann Clin Biochem 1969; 6:24-7.

42. Reitman S, Frankel SA. Colorimetric method for the determination of serum glutamic oxalacetic and glutamic pyruvic transaminases. Am J Clin Pathol 1957; 28:56-63.
43. Campbell WW. DeJong: o exame neurológico. 6a Ed. Rio de Janeiro: Editora Guanabara-Koogan; 2007.

44. Gusmão SS, Campos GB, Teixeira AL. Exame neurológico: bases anatomofuncionais. 2a Ed. Rio de Janeiro: Revinter; 2007.

45. Sanvito WL. Propedêutica neurológica básica. São Paulo: Editora Atheneu; 1996.

46. Auger N, Kofman O, Kosatsky T, Armstrong B. Lowlevel methylmercury exposure as a risk factor for neurologic abnormalities in adults. Neurotoxicology 2005; 26:149-57.

47. Carta P, Flore C, Alinovi R, Ibba A, Tocco MG, Aru $\mathrm{G}$, et al. Sub-clinical neurobehavioral abnormalities associated with low level of mercury exposure through fish consumption. Neurotoxicology 2003; 24:617-23

48. Beuter A, Edwards R. Tremor in Cree subjects exposed to methyl mercury: a preliminary study. Neurotoxicol Teratol 1998; 20:581-9.

49. Tokuomi H. Minamata disease in human adult. In: Kutsuna M, editor. Minamata disease. Kumamoto: Kumamoto University; 1968. p. 37-72.

50. Pacheco-Ferreira H. Mercúrio na Amazônia: efeitos sobre a saúde das populações ribeirinhas [Tese de Doutorado]. Belém: Núcleo de Altos Estudos Amazônicos, Universidade Federal do Pará; 2001.

51. Hamada R, Osame M. Minamata disease and other mercury syndroms. In: Chang LW, editor. Toxicology of metals. New York: CRC Lewis; 1996. p. 337-51.

52. Takaoka S, Kawakami Y, Fujino T, Oh-Ishi F, Motokura F, Kumagai Y, et al. Somatosensory disturbance by methylmercury exposure. Environ Res 2008; 107:6-19.

53. Harada M. Neurotoxicity of methylmercury, Minamata and Amazon. In: Yasui M, Strong MJ, Ota K, Verity MA, editors. Mineral and metal neurotoxicology. Boca Raton: CRC Press; 1997. p. 177-88.

54. Victor M, Ropper AH. Adams and Victor's principles of neurology. 7th Ed. New York: McGraw-Hill; 2001.

Recebido em 28/Out/2012

Versão final reapresentada em 01/Mai/2013

Aprovado em 06/Jun/2013 